\title{
El trabajo social en el sistema educativo vasco, una apuesta social y política
}

\author{
Kristina Jausoro Alzola \\ Delegación Territorial de Educación de Álava, Berritzegune \\ kristina@berrigasteiz.com
}

Edurne Aranguren Vigo

Facultad de Relaciones Laborales y Trabajo Social, Universidad del País Vasco (UPV/EHU)

edurne.aranguren@ehu.eus

\begin{abstract}
Eskola inklusibo baten ideia zuzentasun eta justizia sozialean dago. Hezkuntza ikaskuntza-prozesu integral gisa ulertzen badugu, zaurgarritasuna edo gizarte-bazterkeria egoerak ez lirateke oztopoa izan beharko haurren garapenaren hasierako egoeraren arabera. Horregatik, Euskal hezkuntza-sistemak ezin du alde batera utzi adin txikikoen irteeren desabantailak. Aitzitik, egoera hauek banakako prozesu akademikoak baldintza ez ditzaten bermatzailea izan behar da, irakasleak lagunduz, gizarte eskakizunetara eta konplexutasunera era zientifikoan erantzuteko prestakuntza duten langile adituen bidez. Besteak beste, gizarte langileak dira. Profil ezezaguna, hezkuntzaren esparruan zeresan handikoa, baita beste babes-sistemen arteko elkarreraginean ere. Artikulu honek hezkuntzasistemako gizarte-eskakizun berrien aurrean gizartelangintzaren ikuspegia erakustea du helburu.
\end{abstract}

\section{GAKO-HITZAK:}

Gizarte Langintza, heziketa, justizia soziala, eskola inklusiboa, hezkuntza-sistema, haurren babesa, haurren eskubideak.
La idea de una escuela inclusiva reside en la equidad y la justicia social. Si entendemos la educación como un proceso integral de aprendizaje, las situaciones de vulnerabilidad o exclusión social no deberían ser un obstáculo para que niños y niñas pudieran desarrollarse independientemente de sus situaciones de partida. Por ello, el sistema educativo vasco no puede obviar las desventajas de salida que presentan cada vez más menores. Más bien, debe ser garante de que estas situaciones no condicionen los procesos académicos individuales, apoyando al profesorado a través de profesionales preparadas para responder de una forma científica a las demandas y complejidad social. Entre ellos y ellas se encuentran los y las trabajadoras sociales. Una figura desconocida y con mucho que decir en el ámbito educativo y en su interacción con otros sistemas de protección. Este artículo pretende mostrar la visión del trabajo social ante las nuevas demandas sociales en el sistema educativo.

\section{Palabras Clave:}

Trabajo social, educación, justicia social, escuela inclusiva, sistema educativo, protección infantil, derechos de la infancia. 
La inclusión y la equidad en la educación y a través de ella son la piedra angular de una agenda de la educación transformadora, y por consiguiente nos comprometemos a hacer frente a todas las formas de exclusión y marginación, las disparidades y las desigualdades en el acceso, la participación y los resultados de aprendizaje. Ninguna meta educativa debería considerarse lograda a menos que se haya logrado para todos y todas ${ }^{1}$ (Unesco, 2015).

\section{Las realidades del sistema educativo}

La Declaración de Incheon (Unesco, 2015) supone el punto de arranque en el desarrollo del Plan Marco para el Desarrollo de la Escuela Inclusiva 2019-2020 (Departamento de Educación, 2019) recientemente presentado por el Departamento de Educación del Gobierno Vasco. El objetivo es común, es global, pero plantea las dificultades en su consecución hasta que cada una de las personas menores de edad en proceso de aprendizaje alcance el nivel académico necesario, lo que contribuirá a disminuir en esencia los factores de riesgo de exclusión social y en consecuencia aumentarán los factores protectores para su inclusión plena.

El sistema educativo, en el marco de la escuela inclusiva, se convierte en un facilitador de itinerarios de resiliencia ${ }^{2} y$, en este sentido, en aras de su diversidad, puede dar respuesta a todos y todas implementando los recursos individuales necesarios. También hay que considerar a la escuela como el espacio y contexto idóneo para que todas las diversidades sean visibilizadas y, por ende, acompañadas, apoyadas y si fuese necesario compensadas.

\section{En el citado Plan Marco de Gobierno Vasco} (Departamento de Educación, 2019) se visibiliza un cambio de paradigma que va desde las necesidades educativas especiales (NEES) hasta las barreras para el aprendizaje y la participación, es decir, una apertura a otro tipo de necesidades que no se visibilizan pero que están presentes y dificultan la calidad del aprendizaje de una forma holística. De hecho, se plantea que "los sistemas educativos deben diseñar respuestas flexibles que garanticen el aprendizaje, el logro académico y la inclusión" (pág. 17).

${ }^{1}$ La Declaración para la Educación 2030 se celebró el año 2015 en la localidad de Incheon (Corea), comprometiendo a las diversas naciones a una transformación histórica mediante una nueva perspectiva de la educación. Se concreta en una Agenda de Educación 2030, donde se proponen medidas concretas de intervención. Asimismo, la Declaración de Incheon supuso la semilla de los Objetivos de Desarrollo Sostenible (concretamente el cuarto: Hacia una educación inclusiva, equitativa y de calidad y un aprendizaje permanente para todos y todas), porque encarga a la Unesco, como responsable de la educación en el mundo, la dirección y coordinación de la agenda Educación 2030.

${ }^{2}$ De Dios Uriarte (2006) plantea la resiliencia como aspecto del desarrollo humano que visibiliza las fortalezas y oportunidades que se dan en las personas, los grupos y la sociedad en general, para facilitar a personas que han vivido en contextos desfavorecidos, en situaciones de estrés continuado o de riesgo, un desarrollo con normalidad.
El concepto de necesidad educativa especial ha venido acompañado en la mayoría de ocasiones de un diagnóstico clínico y ha sido tratado como la excepción a la norma. El cambio de paradigma también radica en una visión más abierta para excluir ese carácter de excepcionalidad aportándole una apertura de mirada, el abandono de viejas concepciones y la posibilidad de visibilizar diversidades ocultas ligadas a las historias personales de cada niño y niña, lo cual, hasta ahora, suponía un punto de partida en desventaja para afrontar procesos de aprendizaje.

La comunidad escolar tiene que ser proactiva en esa visibilización respondiendo a las necesidades específicas de cada alumno y alumna. Entre las formas que existen para afrontar la diversidad funcional, existen aquellos modelos que observan la diversidad "desde afuera" como los modelos de exclusión y castigo divino; el tradicional y omnipresente modelo médico-rehabilitador; y modelos que la observan "desde dentro" como los más modernos modelos social o de vida independiente como es el modelo de las capacidades; $y$, por último el modelo de la diversidad, que es donde aparece el término "diversidad funcional" como lo que es: una característica más de "ser humano" (Romañach, 2009) ${ }^{3}$. Realmente, se puede observar un cambio de paradigma, pero, de alguna forma, el sistema sigue categorizando las necesidades educativas en base a unos códigos/etiquetas que establecen la etiología y los apoyos que un alumno o alumna puede recibir en la escuela ${ }^{4}$.

Uno de los retos de la escuela actual podría ser articular una respuesta educativa a los niños, niñas y jóvenes que plantean otro tipo de necesidades por diversas circunstancias, en mayor medida de índole social: situaciones de especial riesgo 0 vulnerabilidad, porque no disponen de un apoyo o de una atención adecuada en el marco familiar, porque viven en situaciones de riesgo y/o desamparo, por ser objeto de maltrato o de acoso, porque son víctimas de violencia de género, por estar tutelados por el sistema de protección, porque sufren ellos o sus adultos problemas de salud mental, por practicar determinadas conductas que ponen en riesgo su salud o la de otras personas, o menores con procesos migratorios en su haber entre otros. Todas y cada una de estas circunstancias tienen un impacto directo en el aprendizaje, en el desarrollo individual de cada persona y su incidencia directa en la dinámica grupal y en la convivencia escolar.

${ }^{3}$ Hay incluso quien refiere que cuando se habla sobre las necesidades específicas no se está hablando tanto de la diversidad funcional, sino más bien de unas “diversidades emocionales”. Este concepto se comentó por parte de Mikel Gotzon Casas Robredo durante una sesión formativa realizada el presente año en los Berritzegunes de Araba destinada al personal docente sobre "El marco legal en la convivencia escolar".

4 Decía George Steiner que "lo que no se nombra no existe”. 
Estas necesidades están detectadas y observadas dentro de la propia escuela. Se puede decir que numéricamente son muy relevantes, aunque no tengan codificación que las haga visibles, o se codifican atendiendo a los síntomas, tal y como sucede con las vinculadas a un diagnóstico clínico o diversidad funcional. Tampoco las cuantifica $y$, sobre todo, no pone en marcha los recursos propios del sistema para poder abordarlos. De alguna forma, el sistema educativo obvia y/o invisibiliza estas necesidades o situaciones de vulnerabilidad. A diferencia de los colectivos vinculados a las diversidades funcionales, que han logrado que el sistema tenga una mirada específica con ellos y articule una respuesta más adecuada, tras la reivindicación colectiva de padres madres y asociaciones del tercer sector; este otro tipo de vulnerabilidades y necesidades no son reivindicadas ni internamente por el propio sistema educativo, ni tampoco por aquellas personas que ejercen la tutela de estos niños, niñas y jóvenes y que, de alguna forma, son los garantes de su protección y tutela $y$, por lo tanto, responsables de su desarrollo. De esta forma, estos niños, niñas y jóvenes quedan en un limbo de desprotección directa e indirecta ante la ausencia de control e interlocución directa que garantice el bienestar integral del alumnado.

\section{La escuela como centro de oportunidades}

Actualmente, el acceso a la educación pública está garantizado en el sistema público vasco desde la etapa infantil, desde los dos años concretamente. Sin embargo, Save the Children (Sastre y Escorial, 2016) considera que, si bien el derecho a la educación contempla el acceso y disfrute en igualdad de condiciones, como las condiciones de partida de cada niña y niño son diferentes, especialmente entre aquellos grupos más vulnerables, para garantizar la igualdad de condiciones se deben poner en marcha medidas compensatorias o correctivas. De hecho, en el citado informe se especifica que "la equidad educativa total se lograría si fuesen los méritos individuales y el esfuerzo del alumnado los únicos factores determinantes de su éxito escolar" (Sastre y Escorial, 2016: 28).

Según el último informe sobre educación realizado por el Consejo Escolar de Euskadi (2018), el índice de escolarización en educación infantil es del $98 \%$ en la población de tres años y del $99 \%$ en la de cuatro años. Se puede decir que el sistema es garantista, pero al observar las tasas de abandono escolar, se puede afirmar que la escuela de hoy en día no ha logrado mantener el vínculo escolar de todo el alumnado y, por lo tanto, garantizar la igualdad de oportunidades. La escuela de hoy en día es un laboratorio de oportunidades, por lo que requiere ser flexible, creativo, dinámico e integrado en la comunidad, trabajando en favor del éxito académico y poniendo énfasis en evitar la desvinculación. Esta desvinculación escolar es "el proceso por el cual el niño o la niña se va alejando de la escuela, emocional, conductual y cognitivamente hasta abandonarla" (Sastre y Escorial, 2016: 31). La tasa de abandono escolar es un constructo estadístico de Eurostat para hacer comparable la calidad de los sistemas educativos y se define como el porcentaje de estudiantes de 18 a 24 años que ha completado, como mucho, estudios de educación secundaria básica (ESO) y no se encuentra en ningún tipo de estudio formación. Según los datos del Ministerio de Educación y Formación Profesional en el año 2018, en el País Vasco el índice de población joven (15 a 29 años) que ni estudia ni trabaja con un nivel de formación inferior a la segunda etapa de educación secundaria es de un 8,2 \% (Ministerio de Educación, 2019).

Si bien es cierto que los índices de abandono escolar en esta comunidad autónoma cumplen los objetivos de la Unión Europea («10 \%), hay un porcentaje significativo de personas para las que la escuela no se ha convertido en el centro de oportunidades deseado ni tampoco en garante de procesos de resiliencia personales que les lleve al logro académico. Qué decir de aquellos centros escolares donde se concentra mayoritariamente alumnado con bajo índice socioeconómico y cultural (ISEC). En estos centros, la segregación es un hecho y tiene una relación directa e influencia en negativo en el rendimiento académico del alumnado. El documento de análisis y propuestas para hacer frente a la segregación escolar en Vitoria-Gasteiz ${ }^{5}$ (Mesa de Trabajo Educación y Diversidad, 2016), es claro en su diagnóstico, y es aquí también donde se deberían aplicar criterios de equidad, no solo facilitando herramientas y recursos para cualquier menor con necesidades educativas específicas en cualquier escuela, sino potenciando programas extraordinarios ya existentes, creando nuevos e introduciendo nuevas figuras profesionales integradas en la comunidad escolar de aquellas escuelas donde el punto de partida es de clara desventaja y dificulta el cumplimiento de los objetivos del Plan Marco de la Escuela Inclusiva marcados por el departamento de Educación. En el propio informe de Save the Children (Sastre y Escorial, 2016), se recomienda diversificar los perfiles profesionales que intervienen en los centros educativos incluyendo en la plantilla trabajadoras y trabajadores sociales, entre otros, dando así una respuesta más integral y cohesionada de toda la comunidad. La cohesión de todos los agentes intervinientes en una escuela (alumnado, familias, profesorado y equipos directivos) es indispensable, así como la cohesión de esta con los diferentes sistemas comunitarios de la ciudad. Estas tareas profesionales están directamente relacionadas con el perfil profesional del trabajo social en cuanto a la labor de cohesión social, de la creación de redes y sinergias.

${ }^{5}$ El 22 de abril de 2016, en sesión ordinaria del pleno del Ayuntamiento de Vitoria-Gasteiz, se aprobó por unanimidad una moción presentada en nombre de la Plataforma a favor de la escuela pública vasca en Vitoria-Gasteiz por una escuela integradora y por una ciudad educadora. 
Esta apuesta por el trabajo social dentro del sistema escolar requiere de un cambio de paradigma pasando del binomio trabajador/a social-servicios sociales, vinculada a las prestaciones económicas, a la idea de un/a profesional que afronta la diversidad y complejidad social mediante instrumentos científicos y procesos de ayuda profesional que facilitan la intervención intra y extra escolar.

\section{El sistema educativo dentro de una galaxia de sistemas: el 'partenariado', más allá de la coordinación}

Una galaxia constituye la agrupación de estrellas, materia cósmica y cuerpos celestes unidas por la atracción gravitatoria, dando como resultado una unidad dentro del universo. Se puede decir que los diversos sistemas de protección constituyen galaxias que son necesariamente interdependientes, atraídas por una fuerza gravitatoria que constituye la ciudadanía y sus derechos y donde ningún sistema puede considerarse independiente 0 autosuficiente. A consecuencia de esta realidad, el propio Ararteko ${ }^{6}$ (2001: 193-194) incluía, entre sus recomendaciones, la necesidad de la coordinación entre diversos agentes (profesionales, servicios, centros, familias, asociaciones, entre otros), mejorando los canales de información, facilitando de esta forma una respuesta global a las necesidades integrales de los y las niñas, jóvenes y sus familias. Pero, además, planteaba la necesidad de una figura clave, dentro de los perfiles profesionales intervinientes, con funciones de seguimiento. De alguna forma, esta figura, que no especifica, podría facilitar la interlocución (intrasistema e intersistemas), el tránsito por las diferentes etapas vitales y escolares, así como la clarificación de las funciones o responsabilidades de cada parte. Esta figura que no se nombra coincide plenamente con las competencias y funciones propias de los y las profesionales del trabajo social. Por todo ello, se explicarán a continuación diferentes niveles de interlocución del sistema educativo.

Dentro del sistema educativo es fundamental la coordinación, dada la diversidad de situaciones que vivencia, así como la diversidad de perfiles profesionales que tiene en su haber. Se elige la coordinación como forma de interlocución clásica en el trabajo social por los siguientes motivos (Fernández Fernández, sin fecha: 50-51):

- El carácter independiente de las problemáticas sociofamiliares que, para el sistema educativo, vienen definidas desde el alumnado con

${ }^{6}$ En este informe específico el Ararteko analiza la respuesta a las necesidades educativas especiales en la Comunidad Autónoma del País Vasco, donde se puede vislumbrar la ausencia de una figura profesional dentro del propio sistema educativo que responda de forma adecuada a las necesidades de los niños, las niñas y sus familias y la inevitable relación con otros sistemas de protección que intervienen necesidades educativas en desventaja o en riesgo de exclusión.

- La transversalidad de las problemáticas sociales del alumnado que requieren atenciones integrales y holísticas, lo que obliga a una relación interinstitucional.

- La adecuación de los recursos y servicios del propio sector educativo. Para el trabajo social los recursos son instrumentos al servicio de otros fines, es decir, al servicio de una mejora continua de los procesos de enseñanza-aprendizaje del alumnado.

- La necesidad de promover y potenciar la convivencia duradera de los servicios de la red, ya que las problemáticas que suelen ser planteadas requieren para su transformación de procesos de intervención simultáneos y prolongados en el tiempo.

El trabajo en equipo dentro del sistema educativo es clave. Para ello, debe haber un reconocimiento de todas las figuras profesionales existentes dentro del sistema. Un trabajo interdisciplinar permite adelantarse a situaciones de conflictividad individuales y/o colectivas que, por lo general, si no se afrontan suelen tener una tendencia a deteriorarse. Es decir, si estas situaciones no se detectan en sus fases iniciales, mediante intervenciones preventivas o reparadoras, suelen agravarse y ocasionar situaciones de desprotección y daños irreparables en el alumnado, en las familias y en los propios centros escolares (Castro Clemente y Pérez Viejo, 2017: 222).

Respecto a la interlocución entre sistemas, el presente artículo defiende, más allá de la coordinación, la cultura del partenariado. Las políticas relativas a la educación no se deciden únicamente en los ámbitos relacionados específicamente con la educación. Es decir, la política educativa está relacionada con las diversas realidades y ámbitos sociales que la cruzan (sanidad, justicia, cultura, bienestar social, entre otras) y de la que son interdependientes. El partenariado es la relación de dos o más organizaciones que se unen para beneficiar a una tercera parte. Cuando se habla de una tercera parte, respecto al sistema educativo y otros sistemas, se podrían integrar a todos y todas las componentes del sistema ${ }^{7}$, pero en este caso el denominador común de esa tercera parte y que se desea traer a colación y visibilizar son los propios niños, niñas y jóvenes. Es decir, una tercera parte que por sí sola, aunque tenga reconocidos unos derechos ${ }^{8}$ como tal, no se puede proteger a sí misma, y por ello es conveniente una figura que la ampare y la proteja de sí misma, de sus iguales, de sus propios progenitores y progenitoras, del profesorado, de los abusos que ejercen algunas personas, es decir, del propio sistema social global. de facto en el cuidado y protección de esos niños y niñas.

\footnotetext{
7 Profesorado, alumnado, familias y asociaciones.

${ }^{8}$ La Convención de los Derechos sobre los Niños y las Niñas (Unicef Comité Español, 2006) y otras leyes estatales que amparan y
} protegen a los niños, niñas y jóvenes. 
El partenariado (Just et al., 2000: 253-258) se da entre distintas organizaciones, de diversa naturaleza9, que se unen para crear algo nuevo o por la confluencia de unos intereses, y que de otra forma no podrían conseguir por sí solas. El partenariado genera sinergias y efectos que multiplican el valor de las acciones de los distintos partners. Es innegable que las necesidades que presentan los niños, las niñas y jóvenes son multidimensionales, por lo que se requiere un abordaje holístico de estas, y "por ser multidimensionales no se solucionan solo ofreciendo recursos" (Just et al., 2000: 256), sino a veces requiere una regulación e intervención integrada en sus diversas dimensiones y liderada por múltiples sistemas. El partenariado, de alguna forma, "cura" el corporativismo, la falsa independencia de los sistemas y la intervención estanca, y aporta compromiso entre sistemas, reciprocidad y la consecución de la cohesión y bienestar social, fin último de la intervención social.

\section{El trabajo social y el sistema educativo, una interacción inevitable}

Hoy en día, en un mundo diverso y globalizado, el sistema educativo afronta nuevas realidades y situaciones donde confluyen las dificultades y carencias en cuanto a recursos financieros y profesionales, y las nuevas demandas emergentes relacionadas con los movimientos migratorios, las situaciones de vulnerabilidad o riesgo de exclusión social, la pobreza económica (desahucios, pobreza energética, ausencia de una alimentación equilibrada, entre otras situaciones), y las diversas formas de violencia entre iguales. Por eso el trabajo social se ubica en diversas dimensiones (individual, familiar, grupal y comunitaria), así como desempeña diversidad de roles adaptándose a una realidad social compleja. Plantea una intervención directa sobre los problemas planteados, pero también se maneja en su rol y función de investigación social para el conocimiento de las causas que generan malestar psicosocial (Zamanillo, 1999). La intervención social con las familias supone uno de los puntos fuertes del trabajo social, ya que la familia, al igual que la escuela, influye en el desarrollo de valores y comportamientos y representa uno de los mejores recursos para mediar y resolver conductas problemáticas (Castro Clemente y Pérez Viejo, 2017: 222).

De fondo existe un debate cada vez más prominente sobre las funciones reales del profesorado en cuanto a la docencia, entendida como actividad profesional consistente en impartir sus clases mediante el cumplimiento de un currículo en una clave de mayoría de alumnado, o si esta figura debe cumplir otro tipo de funciones respecto a la atención de estas situaciones

\footnotetext{
9 De lo contrario, se podría hablar de coordinación cuando se tra-
} ta dentro del propio sistema y se relaciona con la gestión, la organización, la rentabilidad de los esfuerzos y como estrategia para evitar duplicidades en la intervención. de vulnerabilidad o de violencia estructural y directa que cada más vez forman parte del paisaje de la escuela ${ }^{10}$. Pero, más allá de entrar en este debate, cabe indicar que la escuela es el espacio donde más tiempo pasan los niños y las niñas, pero además constituye una microsociedad, por lo tanto, uno de los espacios de socialización y desarrollo mayores en las edades tempranas donde se van a adquirir diversidad de habilidades y capacidades. El alumnado no se puede disociar; más allá de un currículo, tienen unas vivencias fuera de la escuela (a veces muy graves) que van a afectar a su desarrollo intelectual y que es previo. Todo ello va a condicionar su aprendizaje y rendimiento escolar. De esta forma, la escuela se convierte en "un elemento compensador y equilibrante para muchos niños y niñas que atraviesan graves situaciones" (Alemany, 2019).

Las aportaciones y eficacia del trabajo social en el sistema educativo resaltan la importante labor de detectar las necesidades coyunturales de cada momento y época. Su capacidad de adaptar la intervención a cada situación y el amplio conocimiento de primera mano de las problemáticas planteadas representan una combinación de cualidades que provocan la eficacia en la intervención y el refuerzo en la mediación y resolución de diversidad de situaciones del ámbito escolar (Castro Clemente y Pérez Viejo, 2017: 224).

El trabajo social en el sistema educativo es un desconocido; sin embargo, juega un papel fundamental en la parte asistencial y preventiva, que en colaboración con el profesorado facilita intervenir sobre las necesidades, conflictos y problemas que se dan dentro del sistema. Para Torra (2009), no se trata tanto de repartirse los problemas como de actuar conjuntamente para la transformación de estos. Por ello, Castro Clemente y Pérez Viejo (2017) dan un paso más y especifican/explicitan las funciones respecto a cada dimensión de intervención:

- Respecto al alumnado, atender situaciones individuales del estudiante que interfieran en su aprendizaje: absentismo, fracaso educativo, desmotivación, problemas relacionales y de comunicación, inadaptación; percibir conductas conflictivas que perturben la convivencia escolar como el maltrato escolar, acoso o ciberacoso; detectar desajustes familiares: violencia, malos tratos, desprotección o negligencia familiar, deficiencias de recursos básicos, carencias emocionales, falta de higiene, problemas de alimentación, entre otras; prevenir delincuencia y situaciones de inadaptación, facilitar información sobre la situación sociofamiliar del estudiante al centro educativo.

- Respecto al ámbito familiar, potenciar estilos de crianza democráticos, cercanos y comunicativos que promuevan el desarrollo integral de los

${ }^{10}$ Esta realidad se puede constatar por parte de las trabajadoras sociales del berritzegune en su observación diaria y directa en su trabajo directo con los equipos docentes. 
hijos e hijas; colaborar y desarrollar programas de formación, de apoyo, asesoramiento y de orientación familiar a padres y asociaciones de madres y padres; favorecer la participación familiar-comunidad educativa en la marcha del centro; valorar el contexto sociofamiliar de estudiantes con necesidades específicas; implicar a los padres en la búsqueda de soluciones a los problemas que puedan darse en los distintos niveles formativos; fomentar las relaciones familiares entre sí y con el centro educativo.

- En cuanto a la relación con el profesorado, facilitar formación y asesoramiento en coordinación con el centro educativo para dar respuesta a situaciones conflictivas de estudiantes con dificultades sociales procedentes de su contexto familiar y comunitario; proporcionar información sociofamiliar del alumnado; asesorar sobre aspectos que favorezcan el buen funcionamiento del centro y la integración del alumnado con necesidades educativas especiales; participar en la orientación familiar que realiza el profesorado; elaborar información y difundir herramientas y materiales que sean de utilidad al profesorado.

- Y, finalmente, en cuanto al espacio institucional, colaborar en la elaboración del proyecto educativo y en trabajos de investigación sobre necesidades educativas y sociales que posibiliten una adecuada planificación educativa; proporcionar información sobre los recursos sociales y comunitarios y entorno social; establecer y fomentar relaciones fluidas entre la institución y la familia; planificar acciones de prevención y detección precoz de las necesidades educativas especiales; coordinarse y cooperar con los servicios comunitarios existentes para dar respuesta a las necesidades del alumnado: servicios sociales, servicios de protección de menores, de apoyo a mujeres víctimas de violencia y sanitarios.

- Además, orientan, informan y asesoran a otros agentes dentro del sistema educativo (inspección, equipos directivos, entre otros).

Todo ello constituye el quehacer del trabajo social en el sistema educativo, normalmente invisibilizado y desconocido, pero hay literatura al respecto (Fernández Fernández, sin fecha; Colegio Oficial de Diplomados en Trabajo Social y Asistentes Sociales de Madrid, 2007) y múltiples prácticas que deberían ser sistematizadas en investigaciones.

\section{El sistema educativo vasco y el trabajo social, una apuesta social y política}

Hay que indicar, por contextualizar, que el trabajo social en el sistema educativo se inició en el Estado a través de los centros de educación especial mediante la formación de equipos multiprofesionales en los años setenta, aunque actualmente, el trabajo social se ubica en los Equipos de Orientación Educativa y
Pedagógica (EOEP). No existe un criterio unificado en la presencia del trabajo social en los diferentes sistemas educativos, sino que depende de cada comunidad autónoma. En la Comunidad Autónoma de Canarias, por ejemplo, el número de profesionales de trabajo social integrados en los EOEP se eleva a 44 (Consejería de Educación y Universidades, sin fecha). Pero solo hay que dar una vuelta por el globo para comprobar la presencia imprescindible del trabajo social en el sistema educativo (Alemany, 2019):

- Alemania cuenta con profesionales del trabajo social en los centros educativos desde los años setenta ${ }^{11}$.

- Islandia, de la misma forma, cuenta con trabajadores y trabajadoras sociales en los centros escolares desde los años setenta, contando con una gran tradición.

- Respecto a Estados Unidos, muchos colegios e institutos cuentan con profesionales del trabajo social que trabajan la prevención del bullying, del consumo de alcohol, embarazos en la adolescencia, prevención de suicidios, procesos de duelo, etc. ${ }^{12}$.

- En Canadá la figura del trabajo social es muy reconocida en los centros escolares con diversidad de funciones agregadas: apoyo al personal de la escuela, al alumnado y a sus familias, trabajo social con grupos y desarrollo comunitario, prevención del absentismo escolar ${ }^{13}$.

- Australia es un país con gran tradición del trabajo social educativo dado que fue uno de los primeros ámbitos de actuación en que se desarrolló la práctica del trabajo social, existiendo una profesional en cada centro escolar ${ }^{14}$.

- En Chile el trabajo social interviene sobre las situaciones de vulnerabilidad del entorno familiar del alumnado y sobre el que el profesorado no puede intervenir, con una incidencia especial en la participación de las familias en la escuela.

- En México, tras un debate nacional sobre la importancia de la presencia de trabajadoras y trabajadores sociales en los centros escolares como prevención de las brechas y desigualdades entre el propio alumnado, finalmente hace escasos meses el trabajo social fue integrado en la Ley General de Educación ${ }^{15}$.

${ }^{11}$ Sus funciones van desde la prevención de situaciones de riesgo al abordaje de problemas familiares, partiendo de los recursos y fortalezas del alumnado.

${ }^{12}$ Existe un organismo en defensa del trabajo social escolar: The American Council for School Social Work (https://acssw.org).

${ }_{13}$ Los profesionales del trabajo social en el sistema educativo han creado la Canadian Association of School Social Workers and Attendance Counsellors (http://www.casswac.ca/).

${ }_{14}$ Tiene una perspectiva ecológica que abarca todos los ámbitos de referencia del alumnado. Se centra en la prevención de situaciones de riesgo, asesoramiento a todo el sistema, el trabajo grupal y la construcción de redes entre la escuela, la familia y la comunidad.

${ }^{15}$ Los estados de Sonora y Colima ya reconocían anteriormente esta figura en las escuelas. 
- Y, para finalizar con algunos ejemplos a nivel internacional, en Puerto Rico la figura del trabajo social está presente desde 1920 con las maestras visitantes, llegando a 1.800 profesionales en los centros escolares en $2012^{16}$.

La presencia del trabajo social en el sistema educativo vasco no es nueva (Rejado, 2013: 84), pero se puede decir que no es fruto de una reflexión, de una apuesta política, ni de una incorporación de la perspectiva social a la actividad docente. La presencia de los trabajadores y las trabajadoras sociales es casi “anecdótica”, es una rara avis en el conjunto del sistema, fruto de la casualidad del momento histórico. Concretamente, en la actualidad existen tres profesionales, uno en Bizkaia y dos en Araba. El territorio de Gipuzkoa carece de esta figura profesional en el sistema educativo.

En Bizkaia el origen se sitúa en la explosión de gas que sacudió Ortuella el 23 de octubre de 1980. En aquel entonces se incorpora en plantilla un trabajador social en el equipo multiprofesional que el Ministerio de Educación y Ciencia dispuso como apoyo en la gestión de la catástrofe. Con los años, esta figura se integra en el centro de orientación pedagógica (COP, actualmente berritzegune) ${ }^{17} \mathrm{y}$ tras amortizarse dicho puesto de trabajo, en 1995, el trabajador social se incorporó al Centro Escolar Artatse del barrio de Otxarkoaga, ya que desde 1983 dicho centro disponía en su plantilla de ese puesto profesional.

En Araba, por una parte, el origen se remonta a 1974, cuando se contrata a una trabajadora social en el Centro de Educación Especial de Arkaute dependiente de la Diputación Foral de Araba. Este centro se trasladó al Centro Pascual de Andagoya en 1982 y, tras asumir el Gobierno Vasco las competencias en materia de educación especial en 1985 , la figura profesional fue absorbida por el Departamento de Educación. Por otra parte, la Ikastola Umandi de Vitoria-Gasteiz también tenía en su plantilla una trabajadora social y tras realizarse el proceso de publificación esta fue trasladada en 1998 a su ubicación actual en Berritzegune.

Eran los primeros años ochenta, la época de asumir las competencias en materia educativa, y el Departamento de Educación se encontró con que había profesionales del trabajo social adscritos a centros con características particulares específicas, dándose una absorción automática de ese personal. Desde entonces se ha permitido esa presencia, aunque no se ha asumido como una necesidad y en consecuencia no se ha incentivado ni promocionado.

${ }^{16}$ Las funciones que desarrollan están relacionadas con la detección de situaciones de vulnerabilidad social, ofreciendo asesoramiento a la escuela e impulsando las capacidades del alumnado y los derechos de sus familias y del propio alumnado.

${ }^{17}$ En Euskadi, se reconvirtieron en berritzegunes, que se configuran como servicios de apoyo de carácter zonal y/o territorial para la innovación y mejora de la educación en los niveles de enseñanza no universitaria (véase Departamento de Educación, sin fecha).
Por esta razón no ha habido modificación en el número de profesionales y aun siendo únicamente tres en toda la Comunidad Autónoma del País Vasco, representan dos perspectivas/enfoques diferentes de intervención profesional: por un lado, la integrada en el centro de educación infantil y primaria de Artatse en Bilbao, formando parte de la comunidad escolar y con una intervención más directa y cercana con las familias; y, por otro, la que se sitúa en los berritzegunes de Araba, donde se contemplan como figuras de apoyo a las asesorías de necesidades educativas especiales y específicas.

El origen casual de estas figuras profesionales las sitúa en constante cuestionamiento y vulnerabilidad desde las instancias de poder, ya que al tratarse de una presencia no planificada, es fácilmente tachada de innecesaria. No así por parte de los agentes educativos que trabajan con ellas y se sirven de su apoyo y labor profesional. Ante la posible amortización de puestos con un perfil profesional de trabajo social dentro del sistema educativo vasco, el propio sistema plantea una serie de reacciones. Así, en junio de 2018, los equipos directivos de 85 centros escolares de Araba (públicos y concertados) firmaron una petición expresa al Departamento de Educación donde dicen ver imprescindible que

El Departamento de Educación del Gobierno Vasco incorpore en su plantilla profesionales del trabajo social que conozcan la compleja red de servicios sociales, puedan realizar un diagnóstico profesional, asesorar al profesorado y equipos directivos en la identificación de situaciones de riesgo, proponer intervenciones adecuadas, asumir las tareas específicas de interlocución y cohesionar redes interinstitucionales con los mismos referentes profesionales de los servicios sociales en general y los específicos de infancia en particular.

El equipo directivo del CEIP Artatse también ha expresado su preocupación ante la posibilidad de eliminar de la plantilla el perfil del trabajo social, reivindicando su intervención. Las asesorías de NEES de los berritzegunes de Araba se han manifestado en estos mismos términos, solicitando que el departamento tome como ejemplo de buenas prácticas el modelo existente en Araba trasladándose a toda la Comunidad Autónoma del País Vasco. Incluso el Consejo Escolar de Euskadi, en su último dictamen sobre el proyecto de decreto que aprueba la relación de puestos de trabajo del personal laboral no docente de apoyo al alumnado con necesidades educativas especiales en centros docentes de la Comunidad Autónoma del País Vasco, insta al departamento a realizar una evaluación del trabajo de las trabajadoras sociales adecuando la plantilla a las necesidades. El consejo considera también que, dada la situación cada día más complicada en determinados centros, sería necesario incorporar esta figura.

En este escenario de posible desaparición del perfil profesional del trabajo social en el sistema educativo 
vasco, el aumento de la demanda en la intervención social es claro y progresivo. Los equipos docentes se ven desbordados en su día a día por graves dificultades que tienen que ver con situaciones de vulnerabilidad social que necesitan una intervención interdisciplinar y sistémica en continua coordinación y partenariado, que rebasa la competencia docente y que exigen la incorporación de figuras específicas del trabajo social ${ }^{18}$.

En este sentido, los datos aportados en la memoria de los berritzegunes de Araba (A01 y A02) del curso 2017-2018, justifican la necesidad de la intervención social. En el curso 2017-2018 manejaron un total de 1.006 expedientes de alumnado de primaria, secundaria obligatoria y postobligatoria, pertenecientes a 84 centros escolares diferentes tanto de la red pública como de la concertada y podemos afirmar que la práctica diaria rompe de nuevo el mito extendido de que las necesidades existen solo en aquellos centros con bajo índice socioeconómico y cultural. En los casos en que se requiere intervención con niños, niñas y adolescentes pertenecientes a familias con un nivel socioeconómico elevado no usuarias del sistema de servicios sociales, la dificultad aumenta en esa primera intervención al tener que iniciarse en el sistema educativo, procurando y facilitando la incorporación de esas familias a la red de servicios sociales.

Tabla 1. Número de casos según el origen de la demanda

\begin{tabular}{|l|c|}
\hline Origen de la demanda & Número \\
\hline Berritzegune-Asesorías de NEE & 42 \\
\hline Berritzegune y otros & 9 \\
\hline Administración educativa & 15 \\
\hline Centro escolar & 301 \\
\hline Coordinación general & 479 \\
\hline Juzgado & 1 \\
\hline Servicios sociales de base y especializados de Araba & 144 \\
\hline Sistema sanitario & 5 \\
\hline Otros & 10 \\
\hline Total & 1.006 \\
\hline
\end{tabular}

Fuente: Elaboración propia a partir de Ruiz de Aretxabaleta, Jausoro y Rodríguez Álvarez (2018).

Es importante destacar que, atendiendo al origen, el número de demandas directas precedentes del sistema educativo (berritzegune, administración educativa y centros escolares) es de 367 , superando

${ }^{18} \mathrm{El} 21$ de junio de 2017 tuvo lugar, a este respecto, una comparecencia en la Comisión de Educación del Parlamento Vasco donde se expuso la necesidad del trabajo social dentro del sistema educativo de la Comunidad Autónoma del País Vasco (Comisión de Educación , 2017). en gran número a las demandas de intervención iniciadas desde otros sistemas externos (servicios sociales, juzgado, sistema sanitario, otros) que son en total 16o. El número de demandas agrupadas en el apartado de "coordinación general" es de 479. Puede sorprender este dato, ya que no corresponde a una demanda específica sino al número de casos coordinados sistemáticamente con los servicios sociales de base de Araba. En este caso, la demanda es bidireccional entre ambos sistemas. Dado que hay un tercero que se encuentra en situación de vulnerabilidad, la interdependencia entre sistemas es clara y directa.

Nuevamente, se confirma que estas figuras profesionales trabajan desde dentro y para el sistema educativo y de ninguna forma suponen una extensión de los servicios sociales. Sin embargo, supone un nexo de interlocución reconocido dentro del sistema educativo y por parte de otros sistemas de protección.

Atendiendo al motivo de intervención, la casuística es muy diversa y en muchos casos la primera causa de intervención permite sacar a la luz otras muchas dificultades que confluyen en los niños, niñas y jóvenes, pero están invisibilizadas y que necesariamente tienen que ser abordadas.

Tabla 2. Número de casos atendidos y primer motivo de intervención

\begin{tabular}{|c|c|}
\hline Primer motivo de intervención & $\begin{array}{l}\text { Número de } \\
\text { casos }\end{array}$ \\
\hline Absentismo escolar & 70 \\
\hline Abusos sexuales & 18 \\
\hline Comportamiento disruptivo & 59 \\
\hline Comportamiento sexualizado & 4 \\
\hline Consumos & 4 \\
\hline Escolarización/Desescolarización & 54 \\
\hline Instrumentalización & 9 \\
\hline Malos Tratos & 53 \\
\hline Negligencia e incapacidad materna/paterna & 251 \\
\hline Precariedad económica & 34 \\
\hline Tutela & 19 \\
\hline Violencia de género & 28 \\
\hline Otros & 403 \\
\hline Total & 1.006 \\
\hline
\end{tabular}

Fuente: Elaboración propia a partir de Ruiz de Aretxabaleta, Jausoro y Rodríguez Álvarez (2018).

Es destacable o significativo el número de intervenciones cuya primera causa es la incapacidad o negligencia materna y/o paterna, volviendo a evidenciar que en la mayoría de ocasiones las 
intervenciones no están exclusivamente ligadas a la pobreza. Por otro lado, la falta de herramientas técnicas actualizadas y adaptadas que permiten la sistematización de datos y la visibilización de la aparición de nuevas necesidades, hacen que en el concepto de "otros" se agrupen un número elevado de casos sin desglosar sus casuísticas.

Finalmente, hay que señalar que en 2016 el Departamento de Educación puso en marcha el Protocolo de prevención y actuación en el ámbito educativo ante situaciones de posible desprotección y maltrato, acoso y abuso sexual infantil y adolescente y de colaboración y coordinación entre el ámbito educativo y los agentes que intervienen en la protección de la persona menor de edad. Año a año aumentan las notificaciones de ese tipo de situaciones que se hacen desde la escuela al sistema de protección y en la primera mitad del curso 20182019 se han realizado 32 notificaciones oficiales solo en Araba. En todas ellas, a pesar de no estar explícito en el protocolo, se ha requerido desde los diferentes centros escolares la intervención de las trabajadoras sociales del berritzegune en la aplicación de dicho protocolo, realizando el diagnóstico, planificando la intervención con la familia, y en coordinación con los servicios sociales. Todos estos datos aportan evidencias científicas de las intervenciones sociales que tienen lugar dentro del sistema educativo en interdependencia con otros sistemas.

\section{Reflexiones finales}

El trabajo social tiene cabida en todos los sistemas de protección, especialmente en aquellos donde sus sujetos de intervención muestran mayor indefensión, vulnerabilidad y mayor necesidad de protección (nos referimos a las personas menores de edad). La escuela supone un microsistema donde conviven el alumnado, el profesorado y las familias, pero también un espacio de interacción social como ningún otro donde los niños, las niñas y los jóvenes pasan el mayor tiempo de sus vidas cotidianas; $y$, por lo tanto, un observatorio de primer orden para la detección de situaciones que pueden afectar a un desarrollo normalizado. El trabajo social interpela y pone siempre como prioridad a los y las menores. Cabe destacar la responsabilidad pública en la detección del abuso sexual y el maltrato infantil, a veces, obviados por los propios centros escolares ante la incapacidad de reacción, miedo o falta de conocimiento respecto a la respuesta que se debe dar y los pasos a seguir.

El contexto social actual muestra tal complejidad (en parte, por la irrupción de las nuevas tecnologías y las redes sociales), que tanto las familias como el propio profesorado y los equipos directivos se muestran en ocasiones desbordados por las nuevas demandas y situaciones sociales conflictivas o de desprotección a las que se enfrentan cada día. Los y las trabajadoras sociales son el engranaje y la figura profesional referente, que permite el trabajo conjunto y coordinado en la prevención, la detección, el abordaje y la búsqueda de soluciones conjuntas entre los diversos estamentos y sistemas que se ven afectados.

Además, el trabajo social aporta al sistema educativo una mejora en los procesos de resiliencia del alumnado con dificultades, desventajas o en situación de vulnerabilidad, fortaleciendo y empoderando a las familias y a los y las niñas para que puedan afrontar sus procesos de escolarización de una forma normalizada. El trabajo social es una inversión a corto plazo cuando tienen lugar intervenciones en crisis; pero, fundamentalmente, es una inversión a medio y largo plazo, ya que busca una transformación en realidades sociales que dañan y dejan desprotegidos a los y las menores y un bienestar que corresponde por derecho de ciudadanía.

Es clave la incorporación de la figura del trabajo social a los claustros y/o servicios de apoyo para la innovación. Esta presencia mejora la educación desde un punto de vista integral y holístico, reduce la presión que sufre el profesorado, la carga emocional que conlleva, facilitando, al fin y al cabo, su labor docente mediante un acompañamiento cercano y respetuoso. Solo hay que dar una vuelta a nivel internacional para comprobar que este tren hay que cogerlo cuanto antes. Subir a él supone para el sistema educativo, reconocerse interdependiente, complejo y fortalecido, principalmente por la capacidad de mirarse al espejo. Es una apuesta claramente social, técnica y política. El trabajo social ya está en el sistema educativo, solo se trata de conocer qué hace ahí y cómo mejoran las situaciones concretas de las personas a nivel individual, cómo mejoran las familias, cómo mejoran los grupos, cómo mejora la comunidad a través de la escuela y cómo mejora, al fin y al cabo, la propia la escuela. 


\section{Referencias bibliográficas}

ALEMANY, C. (2019): “Por qué necesitamos trabajadores/ as sociales en la escuela", Medium, 7 de abril, <https://medium.com/@carrmen.alemany/ por-qué-necesitamos-trabajadores-as-socialesen-la-escuela-2dc42e94f 5 ccl.

ANECA (2004): Título de grado en Trabajo Social, Serie Libros Blancos, Madrid, Agencia Nacional de Evaluación de la Calidad y Acreditación, «http://www.aneca.es/var/media/150376/ libroblanco_trbjsocial_def.pdf $\%$.

ARARTEKO (2001): La respuesta a las necesidades educativas especiales en la CAPV, Informes Extraordinarios al Parlamento Vasco, VitoriaGasteiz, Ararteko, 〈http://www.ararteko.net/ RecursosWeb/DOCUMENTOS/1/2_586_3.pdf>.

CASTRO CLEMENTE, C. y PÉREZ VIEJO, J. (2017): “El Trabajo Social en el entorno educativo español", Barataria, no 22, pp. 215-226 <https://doi. org/10.20932/barataria.voi22.309'.

COLEGIO OFICIAL DE DIPLOMADOS EN TRABAJO SOCIAL Y ASISTENTES SOCIALES DE MADRID (ed.) (2007): "Trabajo social y educación", no- monográfico extraordinario 1, Trabajo Social Hoy.

COMISIÓN DE EDUCACIÓN (2017): “Comparecencia de trabajadores y trabajadoras sociales de Berritzegune de Vitoria y del CEIP Artatse, a petición de la comisión, de conformidad con la solicitud formulada por Nerea Kortajarena Ibáñez, parlamentaria del grupo EH Bildu, para explicar qué aportaciones puede realizar la disciplina de Trabajo Social a los retos del sistema educativo", Vitoria-Gasteiz, Parlamento Vasco, 〈http://www.legebiltzarra. eus/BASIS/izaro/webx/sm_scomisc/ DDW?W\%3Dsco_serie\%3D5+and+sco_ freun $\% 3$ D20170621+order+by+sco freun\%2Csco_nasun\%26M\%3D5\%26K\%3D201 7062105000005\%26R\%3DY\%26U\%3D1>.
CONSEJERÍA DE EDUCACIÓN Y UNIVERSIDADES (sin fecha): "Distribución de los EOEP", Gobierno de Canarias, 〈http://www.gobiernodecanarias. org/educacion/web/servicios/eoep/ distribucion_eoep/s.

CONSEJO ESCOLAR DE EUSKADI (2018): La educación escolar en Euskadi 2015/2017, Vitoria-Gasteiz, Gobierno Vasco <http://www.consejoescolardeeuskadi. hezkuntza.net/c/document_library/ get_file?uuid=1997a364-6ac8-4518-ba48d93cc4056f9o\&groupld=17937〉.

DE DIOS URIARTE, J. (2006): “Construir la resiliencia en la escuela”, Revista de Psicodidáctica, vol. 11, no 1, pp. 7-24 <http://www.ehu.eus/ojs/index. $\mathrm{php} / \mathrm{psicodidactica/article/view/341>.}$

DEPARTAMENTO DE EDUCACIÓN (2019): Plan Marco para el Desarrollo de una Escuela Inclusiva 20192022, Vitoria-Gasteiz, Gobierno Vasco, 〈https://bideoak2.euskadi.eus/2019/03/07/ news_52628/Plan_Marco_Escuela_Inclusiva. pdf>.

- (2016): Protocolo de prevención y actuación en el ámbito educativo ante situaciones de posible desprotección y maltrato, acoso y abuso sexual infantil y adolescente y de colaboración y coordinación entre el ámbito educativo y los agentes que intervienen en la protección de la persona menor de edad, Vitoria-Gasteiz, Servicio Central de Publicaciones del Gobierno Vasco, 〈http://www.euskadi.eus/contenidos/ informacion/dif11/es_5613/adjuntos/ Protocolo_Educacion_15_noviembre_2016.pdf〉.

- (sin fecha): Berritzeguneak, 〈http://www.berritzeguneak. net>.

ESTIVILL PASCUAL, J.; HIERNAUX, J. y GEDDES, M. (1997): El partenariado social en Europa, Barcelona, Hacer. 
FERNÁNDEZ FERNÁNDEZ, D. (sin fecha): El Trabajo Social en el Sistema Educativo, Consejo General del Trabajo Social, «https://www.cgtrabajosocial. es/files/52bc5b6a6cada/EL_TRABAJO_SOCIAL_ EDUCATIVO.pdf〉.

JUST, J.; MARTÍNEZ, O.; ESTANYOL, V. y MORA, A. (2000): "El partenariado como estrategia del trabajo social comunitario", Cuadernos de Trabajo Social, no 13 , pp. 253-268, <https://revistas. ucm.es/index.php/CUTS/article/view/ CUTS0000110253A>.

MESA DE TRABAJO EDUCACIÓN Y DIVERSIDAD (2016): Documento de análisis y propuestas para hacer frente a la segregación escolar en VitoriaGasteiz, 19 de diciembre, 〈https://blogs.vitoriagasteiz.org/medios/files/2017/03/Documentodefinitivo-segregación-escolar.pdf〉.

MINISTERIO DE EDUCACIÓN Y FORMACIÓN PROFESIONAL (2019): Enseñanzas no universitarias [estadísticas], Ministerio de Educación y Formación Profesional, <http://www. educacionyfp.gob.es/servicios-al-ciudadanomecd/estadisticas/educacion/no-universitaria. html.

REJADO, M. (2013): Inicios y consolidación del trabajo social en Álava, Vitoria-Gasteiz, Universidad del País Vasco.

ROMAÑACH, J. (2009): Bioética al otro lado del espejo. La visión de las personas con diversidad funcional y el respeto a los Derecho Humanos, A Coruña, Diversitas, Asociación de Iniciativas y Estudios
Sociales, <http://www.diversocracia.org/docs/ Bioetica_al_otro_lado_del_espejo_v_papel. pdf>.

RUIZ DE ARETXABALETA, K.; JAUSORO, K. y RODRÍGUEZ ÁlVAREZ, V (2018): Memoria Curso 2017-2018 Trabajadoras Sociales A01-Ao2 Berritzeguneak, Vitoria-Gasteiz, Gobierno Vasco.

SASTRE, A. y ESCORIAL, A. (coords.) (2016): Necesita mejorar. Por un sistema educativo que no deje a nadie atrás, Save The Children España, <https://www.savethechildren.es/sites/ default/files/imce/docs/necesita-mejorarfracaso-escolar-savethechildren-ok.pdf〉.

TORRA, M. (2009): “El Trabajo Social en la enseñanza”, Revista de Servicios Sociales y Política Social, no 86, pp. 125-128.

UNESCO (2015): Education 2030: Incheon Declaration and Framework for Action for the Implementation of Sustainable Development Goal 4: Ensure Inclusive and Equitable Quality Education and Promote Lifelonglearning Opportunities for All, United Nations Educational, Scientific and Cultural Organization, 〈https://unesdoc. unesco.org/ark:/48223/pfooo0245656>.

UNICEF COMITÉ ESPAÑOL (2006): Convención sobre los Derechos de los Niños y las Niñas, Madrid, Nuevo Siglo.

ZAMANILLO, T. (1999): “Apuntes sobre el objeto de trabajo social”, Cuadernos de Trabajo Social, nํㅜㄹ, pp.12-32. 